\title{
EL CONCEPTO DE DERECHO: UNA NUEVA APROXIMACIÓN METODOLÓGICA A LA TEORÍA DE ROBERT ALEXY*
}

\author{
Paula Gaido ** \\ Universidad Nacional de Córdoba (Argentina)
}

RESUMEN. En este trabajo me propongo clarificar cuál es la idea que subyace a la afirmación de $\mathrm{R}$. ALEXY de que el propósito de la Filosofía del Derecho es dar una explicación de la naturaleza del Derecho. En este contexto la pregunta por la naturaleza del Derecho es una pregunta por sus propiedades esenciales, esto es, por sus propiedades necesarias. Identificar cómo se entiende la idea de lo necesario es entonces central. Esta expresión es usada de manera ambigua por ALEXY, lo cual impacta en su uso también ambiguo de la expresión "concepto de Derecho". Sugiero que usa la expresión "concepto de Derecho" en dos sentidos, y mantengo que, si esto se tiene en cuenta, se puede identificar mejor la empresa teórica con la cual está en realidad comprometido. Finalmente, señalo una contradicción adicional en sus argumentos cuando afirma que la naturaleza del Derecho sólo puede ser captada desde el punto de vista interno.

Palabras clave: concepto de Derecho, naturaleza del Derecho, punto de vista interno, R. AleXY.

ABSTRACT. In this paper I try to make clear R. ALEXY's idea when he says that the purpose of the philosophy of law is to give an explanation of the nature of law. In this context the question about the nature of law is a question about its essential properties, that is, about its necessary properties. To identify how the idea of necessity is understood is thus central. ALEXY uses this expression ambiguously, and that impacts his use of the expression "concept of law», which is also ambiguous. I suggest that he uses the expression the "concept of law» in two different senses, and maintain that if that is taken into account, we could better identify which is the theoretical enterprise with which ALEXY is really committed. Finally, I notice a further contradiction in his argument when he affirms that the nature of law can be captured only from the perspective of the participants of the legal practice.

Keywords: the concept of law, the nature of law, the internal point of view, R. ALEXY.

* Fecha de recepción: 31 de julio de 2009. Fecha de aceptación: 3 de septiembre de 2009.

** Expuse una primera versión de este trabajo en el DAAD-Alumni-Veranstaltung, Fachseminar «Grundrechte, Diskurs und praktische Vernunft. Die Rechtstheorie R. Alexy's», 7-9 de octubre de 2008, Universidad de Buenos Aires, organizado por L. Clérico e I. SiecKMAnN, donde R. AleXXY estuvo presente. Tal versión fue publicada como «The Concept of Law: A Methodological Approach to Alexy's Theory», en L. CLÉRICO e I. SiECKMAnN (eds.), Grundrechte und Diskurs, Baden-Baden, Nomos, 2009. La discusión que tuvo lugar en ese momento me ha llevado a revisar algunos de los argumentos que corrijo con esta presentación. Por otra parte aquí incorporo otras consideraciones que en aquella primera exposición dejé de lado. Quiero agradecer a R. CARACCIOLO y C. REDONDO sus críticas a las versiones preliminares de este escrito. 




ara ALEXY el propósito de la Filosofía del Derecho es dar una explicación de la naturaleza del Derecho ${ }^{1}$. Y, en este contexto, una pregunta por la naturaleza del Derecho es una pregunta por sus propiedades esenciales; o, dicho en otras palabras, por aquello que hace que el Derecho sea lo que es, y no otra $\operatorname{cosa}^{2}$. Cuando ALEXY se refiere a las propiedades esenciales del Derecho se está refiriendo a sus propiedades necesarias. Considero central, entonces, elucidar la manera en que ALEXY entiende la idea de lo necesario, ya que éste es un presupuesto básico en la articulación de los compromisos filosóficos de cualquier concepción teórica. Lo primero que detecté al encarar esta tarea, es el carácter ambiguo de la noción de lo necesario en su teoría. Aquí lo que está en cuestión es elucidar si la idea de lo necesario depende de cuáles sean consideradas propiedades necesarias por ciertas comunidades culturales o si el carácter necesario de tales propiedades es independiente de toda comunidad cultural. En lo que sigue intentaré establecer las diferencias en la manera de entender lo necesario, para así precisar cuál es la noción relevante en juego y, junto a ella, su idea de propiedad esencial. Esto permitirá, a su vez, esclarecer cómo entiende AlEXY la tarea filosófica en el ámbito jurídico.

\section{IDEA DE «NECESIDAD» EN JUEGO}

Para comenzar hay que apuntar que ALEXY distingue cuatro tipos principales de «necesidad»: relativa, absoluta, conceptual y normativa ${ }^{3}$. Una necesidad es relativa cuando los límites de lo necesario son fijados por un determinado esquema conceptual o una práctica social, que puede variar. Una necesidad es absoluta, por el contrario, cuando los límites de lo necesario son fijados por un esquema conceptual o práctica social, que es inmune a la revisión ${ }^{4}$. Como primera precisión, cabe señalar que, la argumentación de ALEXY acerca de cuáles son las propiedades esenciales del Derecho se desarrollan asumiendo como presupuesto que lo que está en cuestión es una necesidad relativa $^{5}$. No se trata de averiguar cuáles son las propiedades necesarias del Derecho

1 R. AleXY, «The Nature of Legal Philosophy», Ratio Juris, vol. 17, núm. 2, 2004, p. 156 (en adelante lo citaré como NLPh), R. ALEXY, «The Nature of Arguments about the Nature of Law», en L. MEYER, S. PAULSON y T. Pogge (eds.), Rights, Culture, and the Law. Themes from the Legal and Political Philosophy of Joseph Raz, Oxford, 2003, p. 4 (en adelante lo citaré como NANL).

2 En esta línea, ALEXY sostiene: «...existe un conjunto de características que todos los sistemas jurídicos y todo Derecho deben poseer para ser un sistema jurídico o Derecho, con independencia de todo tiempo y espacio, ...hay ciertas propiedades que son necesarias para ser un sistema jurídico o Derecho, esto es, ciertas universalia iuris (P.G.)», en R. ALEXY, «Law, Discourse and Time», Archiv für Recht und Sozial Philosophie, 1995, pp. 101-102.

3 ALEXY utiliza de manera indistinta las expresiones «necesidad normativa»o «necesidad práctica». Aquí hablaré sólo de necesidad normativa para simplificar la exposición.

${ }^{4}$ En palabras de ALEXY: «La contrapartida de la necesidad relativa es la necesidad absoluta. La necesidad absoluta implica la inmunidad de un esquema conceptual frente a la revisión. La necesidad relativa no es una necesidad del esquema conceptual sino una necesidad dentro del esquema conceptual», R. ALEXY, NANL, p. 8, nota 17 (cito de aquí en adelante la versión en castellano de C. BERNAL, «La naturaleza de los argumentos sobre la naturaleza del Derecho», en R. ALEXY, El concepto y la naturaleza del Derecho, Madrid, Marcial Pons, 2008; mantengo, sin embargo, la paginación de la versión original). Aunque aquí ALEXY tan sólo hace referencia a la noción de esquema conceptual, considero que la idea es extensiva a la noción de práctica social.

5 ALEXY sostiene que busca, al asumir este compromiso, hacer frente a la objeción planteada por Quine, dirigida, dicho de manera gruesa, a impugnar la posibilidad de un análisis conceptual último en términos de condiciones necesarias y suficientes. ALEXY, en este sentido, dice: «La necesidad dentro de un específico esque- 
de acuerdo con un esquema conceptual o práctica social inmune al cambio, sino de su individualización tomando como punto de partida ciertos esquemas conceptuales y prácticas sociales no inmunes al cambio. Es con este recorte con el que entran a jugar en su teoría las nociones de necesidad conceptual y necesidad normativa.

Una necesidad es conceptual cuando depende de las convenciones lingüísticas de una determinada práctica, dentro de las cuales están incluidas, en el presente contexto, las reglas constitutivas de los respectivos actos lingüísticos. Para averiguar cuáles son las propiedades necesarias del Derecho, desde esta perspectiva, hay que recurrir, en primer lugar, al uso del lenguaje, esto es, al análisis de las reglas semánticas vigentes que fijan el significado del término «Derecho» ${ }^{6}$. A su vez, hay que recurrir al análisis de las reglas constitutivas de los actos de habla, ya que ellas determinan cuáles son los presupuestos requeridos para que los actos jurídicos, en cuanto actos de habla específicos, puedan verificarse, lo cual tiene impacto en la manera de pensar al Derecho. Así, dado que las convenciones lingüísticas pueden variar, también pueden hacerlo las propiedades que según ellas sean necesarias ${ }^{7}$. Por último, cuando una necesidad es normativa lo necesario está vinculado con los valores subyacentes a la práctica jurídica. AlEXY entiende que los actos jurídicos, en tanto que actos de habla regulativos, están comprometidos de manera necesaria con la afirmación de ciertos valores. Es así como, desde una perspectiva normativa, lo necesario está relacionado con los valores subyacentes a la práctica jurídica entendida como práctica lingüística regulativa, esto es, dirigida a decidir sobre aquello que es jurídicamente debido ${ }^{8}$. El compromiso con estos valores está intrínsecamente unido a aquello que constituye las prácticas lingüísticas regulativas en general ${ }^{9}$, de manera tal que, habría que concluir, si cambian estas prácticas, el compromiso con estos valores puede desaparecer, y junto a ello aquello que es definitorio del Derecho. Cabe agregar que, entre lo que es normativamente necesario, AlEXY distingue, a su vez, entre un sentido débil y un sentido fuerte. La diferencia entre los diferentes tipos de necesidad normativa depende, respectivamente, de si los valores en cuestión son relativos a consideraciones fácticas o si, en cambio, son requeridos por la razón práctica ${ }^{10}$.

ma conceptual o sistema, y también dentro de una práctica específica, está en oposición a la necesidad de un específico esquema conceptual, sistema o práctica en cuanto tal, y es consistente con la tesis de Quine que dice que ninguna oración o regla es inmune a la revisión (P.G.)», R. ALEXY, «My Philosophy of Law: The Institutionalisation of Reason», en L. Witgens (ed.), The Law in Philosophical Perspectives, Dordrecht-Boston-London, Kluwer Academic Publishers, 1999, p. 27.

${ }^{6}$ Cfr. R. AleXY, «On the Concept and the Nature of Law», Ratio Juris, vol. 21, núm. 3, septiembre 2008, p. 292 (en adelante lo citaré como OCNL). Aquí es preciso señalar que en numerosas oportunidades ALEXY utiliza las expresiones «concepto» y «significado» como sinónimas. Esto se considera un defecto ya que oscurece las distintas ideas que están subyacentes, y que ALEXY mismo está interesado en diferenciar. Retomaré este punto más adelante.

7 Tal como lo afirma ALEXY: «El uso del lenguaje puede cambiar. Es por esta razón que me he referido al concepto de Derecho "tal como es usado en el presente" », R. ALEXY, NANL, p. 8. Con relación al cambio de las reglas constitutivas de los actos jurídicos como actos de habla específicos, cfr. R. ALEXY NANL, pp. 12-13; R. ALEXY, «My Philosophy of Law...», op. cit., p. 27.

8 Cfr. R. Alexy, NANL, pp. 8-10.

9 Existen diferencias relevantes en la propuesta de ALEXY en relación a las prácticas regulativas en el ámbito jurídico. No es mi intención profundizar en este trabajo sobre este punto.

10 ALEXY se refiere al carácter ambiguo de la noción de necesidad normativa en los siguientes términos: «El concepto de necesidad práctica es ambiguo. Una interpretación débil únicamente se refiere a un tipo de relación medio-fin, en la que la elección del fin se trata sólo como un asunto fáctico o únicamente como algo 
En virtud de lo dicho, para elucidar cuál es la idea que está en juego de lo necesario en el análisis de las propiedades del Derecho que ALEXY considera esenciales, cabe concluir que son argumentos conceptuales y normativos los que el autor afirma que están implicados en su explicación ${ }^{11}$. Los argumentos conceptuales están dirigidos al análisis del término «Derecho» y de la estructura de los actos jurídicos, tales como legislar o juzgar, en tanto que actos de habla regulativos. De acuerdo con lo antes establecido, hay que concluir que son estos argumentos los que están orientados a la determinación de las propiedades del Derecho conceptualmente necesarias. Los argumentos normativos, por otra parte, tienen como propósito ofrecer la mejor versión de la práctica jurídica, a la luz de sus valores subyacentes ${ }^{12}$. En este sentido, hay que concluir que están dirigidos a la determinación de las propiedades del Derecho normativamente necesarias. Si se tiene en cuenta la distinción trazada en el párrafo precedente, hay que adelantar que ALEXY toma el sentido fuerte de lo «normativamente necesario» en la determinación de las propiedades esenciales del Derecho.

Teniendo en cuenta la relevancia de los argumentos conceptuales y normativos en la explicación de la naturaleza del Derecho, es posible llegar a algunas conclusiones preliminares. En primer lugar, la idea de necesidad en juego es relativa y, en este sentido, cuáles sean las propiedades esenciales del Derecho depende del análisis de un esquema conceptual en particular o práctica social, que puede variar ${ }^{13}$. En segundo lugar, las consideraciones relevantes en la elucidación de las propiedades esenciales del Derecho serán no sólo meramente conceptuales, sino normativas ${ }^{14}$. La vía elegida por ALEXY para dar cuenta de cuáles son las propiedades esenciales del Derecho incluye una articulación de los compromisos valorativos implicados en la manera de entender el Derecho. La comunidad de referencia relevante, sin embargo, no es la constituida por los participantes de una específica práctica jurídica (por ejemplo, la propia de la sociedad moderna occidental) ${ }^{15}$, sino la constituida por

hipotético. Esta es la trascendencia del concepto de HART de "necesidad natural", si los fines se entienden sólo como "algunas generalizaciones muy obvias... concernientes a la naturaleza humana y al mundo en que el hombre vive". No obstante, el panorama comienza a cambiar si los fines generales del Derecho, como la certeza jurídica y la protección de derechos fundamentales, son considerados como exigencias de la razón práctica, y cambia completamente, si estas exigencias son consideradas como elementos necesarios del Derecho. Una interpretación fuerte como esta del concepto de necesidad práctica constituiría una base normativa o evaluativa del Derecho», R. AlEXY, NLPh, p. 165.

${ }_{11}$ Cfr. R. AleXY, NANL, pp. 6-7. AlEXy habla de manera indistinta de «argumentos normativos» y «argumentos prácticos». Utilizaré tan sólo la primera expresión para simplificar la exposición.

${ }^{12}$ Cfr. R. Alexy, NANL, pp. 7-8.

13 Esta idea está subyacente en la siguiente afirmación de ALEXY: «El carácter relativo de la pretensión de corrección a una práctica específica coincide en su estructura con la tesis de GRICE y STRAWSON de que una cosa es admitir que no existe una necesidad absoluta para adoptar o usar esquema o sistema conceptual alguno, y otra distinta decir que no hay necesidades dentro de cualquier esquema o sistema conceptual que adoptamos o usamos (P.G.)», R. AleXY, «My Philosophy of Law», op. cit., nota 10, p. 27. En esta clave afirma: «La pretensión de corrección, por tanto, sólo es necesaria dentro del marco de una práctica específica; como consecuencia, la necesidad en cuestión es sólo una necesidad relativa. Antes se ha mencionado que un «deber ser» que sea más que una expresión de voluntad sólo puede definirse por medio de un concepto de corrección», R. ALEXY, NANL, p. 13.

${ }_{14}$ Más adelante, como se verá, lo normativo es, para ALEXY, conceptualmente necesario.

${ }_{15}$ Como, por ejemplo, sí lo es para J. RAZ, $c f r$. J. RAZ, «Puede haber una teoría del Derecho?», trad. R. SÁnchez Brigido, en H. Bouvier, P. GAIDO, R. SÁNChez BRIgIDo (eds.), Una discussion sobre teoría del Derecho, Madrid, Marcial Pons, 2007, pp. 47-86 [título original: «Can there be a Theory of Law», en GoLDING y 
quienes adoptan la perspectiva del participante respecto del Derecho, sin importar su pertenencia institucional ${ }^{16}$.

AlEXY considera que el propósito de la teoría del Derecho es explicar la naturaleza del Derecho, y que es exitosa si consiste en un conjunto de proposiciones sobre el Derecho necesariamente verdaderas, que explican lo que el Derecho verdaderamente es. Sin embargo, lo que el Derecho verdaderamente es parece estar intrínsecamente vinculado con lo que el Derecho debe ser en términos ideales. Esta conclusión resulta inevitable si se tiene en cuenta que, para ALEXY, aun cuando los argumentos conceptuales son importantes en la explicación de la naturaleza del Derecho, son los argumentos normativos los que tienen la última palabra ${ }^{17}$. Pareciera que AlEXY está pensando que aquello que el Derecho verdaderamente es se encuentra latente en nuestras prácticas lingüísticas vigentes, y que es tarea del teórico del Derecho intentar la articulación de su forma perfecta. Así, lo que el Derecho verdaderamente sea no es algo que de manera necesaria exista en el mundo tal cual es, ni que se encuentre reflejado en el uso contingente que se hace del término «Derecho», ni que pueda proponer el teórico sin tener de alguna manera en cuenta a las prácticas existentes. Más bien, como lo indiqué, parece estar implicado en los compromisos valorativos subyacentes a la práctica jurídica en tanto que práctica lingüística de especiales características. La naturaleza del Derecho estaría relacionada con los valores subyacentes a esta práctica y, de esta forma, con un mundo ideal. Para ALEXY, entonces, las propiedades esenciales del Derecho no serían equivalentes a las propiedades asociadas al Derecho de manera contingente por la práctica de un lenguaje, sino a ciertas propiedades finitas que tiene el Derecho en tanto que práctica regulativa comprometida por ciertos valores, de los cuales — cabe conjeturar - se puede no tener un manejo acabado o dominio completo.

Hasta aquí pareciera que explicación del concepto de Derecho y explicación de la naturaleza del Derecho difieren. El análisis conceptual —en cuanto análisis semántico y análisis pragmático del lenguaje- forma tan sólo parte de la explicación de la naturaleza del Derecho. Sin embargo, cuando AlEXY articula su concepción acerca de los conceptos, se advierte que el análisis conceptual —en los términos aquí desarrollados- también es considerado tan sólo una de las herramientas teóricas disponibles en la explicación de los conceptos. Su manejo ambiguo del término «concepto» hace que, sin precisiones adicionales, no sean transparentes las diferentes ideas en cuestión. Será necesario despejar esta ambigüedad para ver hasta qué punto la explicación de la naturaleza del Derecho y la explicación del concepto de Derecho difieren. A esta tarea dedicaré el próximo apartado.

EDmunson (eds.), The Blackwell Guide to Philosophy of Law and Legal Theory, Georgia State Univ., Blackwell Publishers, 2004, pp. 324-342].

${ }_{16}$ Vuelvo enseguida sobre este punto.

17 En esta línea ALEXY señala: «El análisis del uso del lenguaje, como J. L. AusTiN acertadamente señaló, no es ciertamente "la última palabra", pero provee un punto de partida para el análisis o — como Austin lo expresó- una "primera palabra". Debo intentar confirmar esta idea mediante la presentación de un argumento conceptual como primera palabra y un argumento práctico o normativo como la última», R. ALEXY, NANL, p. 7; en la misma línea $c f r$. R. AlEXY, NLPh, pp. 158-159. 


\section{LA NOCIÓN DE «CONCEPTO» EN JUEGO}

Tal cual señalé, un análisis conceptual del Derecho —en los términos anteriormente descritos- no es considerado por ALEXY una explicación completa de la naturaleza del Derecho, sino parcial. Frente a la pregunta de si, en cambio, tal análisis puede ser considerado una explicación del concepto de Derecho, advierto con sorpresa que la respuesta de AlEXY es negativa. Para poder hacer inteligible su posición, es preciso disolver las ambigüedades señaladas en el apartado anterior con relación al uso que realiza de la expresión «concepto de Derecho» en sus diferentes escritos. Aclarar este punto, a su vez, permitirá comprender la manera en que ALEXY entiende la tarea filosófica, y, en particular, cuál es el lugar que le asigna al análisis conceptual. Para ello analizaré los siguientes apartados:

\subsection{Conceptos vigentes y conceptos correctos}

ALEXY parece interesado en distinguir entre diferentes conceptos: vigentes y correctos. El término «vigente» no es empleado por AlEXY en sus escritos. Sin embargo, en el análisis de su teoría lo utilizaré para distinguir una de las ideas en cuestión cuando el autor usa el término «concepto». En reiteradas oportunidades AlEXY utiliza de manera indistinta los términos «significado» y «concepto», lo cual conduce a pensar que su uso intercambiado no implica una diferencia en la idea que se quiere transmitir. Esto haría concluir que no está especialmente interesado en trazar una distinción entre ambos términos. Sin embargo, ello constituiría un error si lleva a pensar que es siempre la misma idea la que está en consideración cuando se utiliza el término «concepto». La misma idea estaría en cuestión con la utilización de los términos «significado» y «concepto vigente», pero no así cuando habla de «concepto correcto». AlEXY, sin embargo, emplea el término «concepto» sin más aditamentos para referirse a ambas ideas, lo cual a veces opaca aquello a lo que apuntan sus argumentos. El hecho de que dos son las ideas en cuestión se ve con claridad cuando expone cuál es su concepción sobre los conceptos. Vale la pena, entonces, detenerse en su exposición.

Para AlEXY los conceptos son entidades complejas, en parte locales y en parte universales. Con ello, busca fijar una posición intermedia entre quienes entienden a los conceptos o bien como entidades sociales, o bien como entidades ideales independientes de todo origen social. En esta línea, ALEXY afirma que los conceptos tienen dos dimensiones: una convencional, vinculada a las reglas sociales que establecen el significado de las palabras; y otra ideal, vinculada a una pretensión de adecuación de los conceptos a los objetos a los que se refieren ${ }^{18}$. En este último caso, el término «concepto»

18 Las palabras de ALEXY son claras al respecto: «El concepto de Derecho tiene naturaleza local en cuanto que su posesión depende de la pertenencia a una cierta cultura o, por lo menos, a estar familiarizado con ella. Los conceptos son entidades complejas que comprenden más de una dimensión. Como productos de una cultura, son reglas socialmente establecidas que involucran el significado de las palabras. Hasta aquí, la naturaleza "local" de los conceptos implica su carácter convencional. Pero los conceptos —y éste es el otro lado de su naturaleza - son convenciones o reglas de un tipo especial. Ellos pretenden ser "adecuados a su objeto". De esta manera, están intrínsecamente relacionados a la corrección o verdad de las proposiciones construidas 
tiene que ser entendido como «concepto vigente». Las convenciones lingüísticas que sustentan a estos «conceptos vigentes», afirma ALEXY, tienen una naturaleza especial ya que, de manera necesaria, pretenden adecuarse a los objetos a los que se refieren; o lo que sería lo mismo, pretenden aprehender su naturaleza. Esto quiere decir que, los conceptos, entendidos como reglas convencionales del uso de las palabras, serán objetivamente correctos si reflejan la esencia o naturaleza de los objetos a los que se refieren ${ }^{19}$. Aquí lo que quiero destacar es que, para ALEXY, los conceptos de Derecho «vigentes» serán conceptos «correctos» o «incorrectos» de Derecho, según logren satisfacer la pretensión de adecuación por ellos formulada, o no. En este sentido, es posible interpretar a ALEXY en clave kantiana, y sostener que entiende al concepto de Derecho correcto como una suerte de idea regulativa subyacente en las prácticas que sustentan los diferentes conceptos vigentes de Derecho ${ }^{20}$. Así, mientras que, para ALEXY, sería posible afirmar la existencia de diferentes conceptos vigentes de Derecho, no parecería posible realizar la misma afirmación respecto de la existencia de más de un concepto correcto de Derecho. Si la naturaleza del Derecho es una, de la misma manera tiene que ser uno el concepto de Derecho que la refleje. Sólo así puede ser entendida la afirmación de ALEXY que sostiene que el concepto correcto de Derecho tiene validez universal ${ }^{21}$.

por medio de ellos. Esta pretensión de adecuación conecta necesariamente el concepto de una cosa con su naturaleza. Los conceptos - como partes de una práctica que está intrínsecamente conectada con la verdad, justificación, validez intersubjetiva, objetividad y realidad - procuran captar la naturaleza de las cosas a las que ellos se refieren tan perfecta o correctamente como sea posible. Esta es la dimensión no convencional o ideal de los conceptos», R. ALEXY, «Acerca de dos yuxtaposiciones: concepto y naturaleza, Derecho y filosofía. Algunos comentarios sobre "¿Puede haber una teoría del Derecho? de Joseph Raz"», trad. P. GAIDO, en Una discusión sobre teoría del Derecho, op. cit., p. 90 (título original: «On Two Juxtapositions: Concept and Nature, Law and Philosophy. Some Comments on Joseph Raz's "Can There Be a Theory of Law?"», Ratio Juris, vol. 20, núm. 2, 2007, pp. 162-169).

19 Esta es la idea de ALEXY cuando sostiene: «Los conceptos basados en el uso actual del lenguaje necesitan ser modificados, cuando se prueba, como dice KANT — quien menciona, entre otros, los conceptos de oro, agua y Derecho- que no son "adecuados a su objeto"», R. ALEXY, NLPh, p. 163.

${ }^{20}$ En esta idea parece estar pensando ALEXY cuando afirma: «Es una pregunta abierta si es que alguna vez se pueda lograr congruencia perfecta alguna. Pero como idea regulativa guía nuestra práctica de usar conceptos» (el énfasis me pertenece), R. ALEXY, «Acerca de dos yuxtaposiciones...», op. cit., p. 91. Otra alternativa posible, sería relacionar la perspectiva de ALEXY con la noción de conceptos en la línea de las semánticas K-P. Sin embargo, para ALEXY los conceptos encuentran sus raíces en la realidad social, pero que alojan su patrón de corrección en una realidad «ideal». Es así como, aun cuando ALEXY sostiene la relevancia de las convenciones, ubica aquello hacia lo que tienden en un espacio externo, pero no ya físico - como el propuesto por las semánticas K-P_, sino ideal. La diferencia central con las semánticas $\mathrm{K}-\mathrm{P}$ consistiría en que no es nada en un mundo externo lo que causa la manera que en nuestras prácticas tenemos de entender al Derecho. Más bien es a la inversa. Es el trasfondo de nuestras convenciones lo que nos conduce a que, para articular de manera completa lo que pensamos, tengamos que recurrir a un mundo valorativo o ideal. En esta clave AlEXY sostiene: «El concepto de Derecho se refiere a una entidad que conecta lo real con lo ideal de una manera necesaria. A pesar de su anclaje en el mundo real, el Derecho no puede ser reducido a una clase natural o a un objeto, tal como ocurre con los conceptos de agua, agujeros negros o matar. El concepto de Derecho representa un paradigma de un concepto de una clase no-natural que está intrínsecamente ligado con clases naturales», R. ALEXY, OCNL, p. 284 (cito de aquí en adelante la traducción de C. BERNAL, «En torno al concepto y la naturaleza del Derecho», en R. ALEXY, El concepto y la naturaleza del Derecho, Madrid, Marcial Pons, 2008; mantengo, sin embargo, la paginación de la publicación original).

${ }^{21}$ Cfr. R. AlEXY, «Acerca de dos yuxtaposiciones», op. cit., p. 90. 


\subsection{Concepto, objeto y propiedades esenciales}

Es importante advertir un dato adicional que, en principio, resulta contradictorio respecto de los compromisos meta-teóricos que considero estructuran la teoría de ALEXY. La cuestión aludida se refiere a que, para AlEXY, no es el análisis de cualquier concepto vigente de Derecho el relevante en la explicación de la verdadera naturaleza del Derecho. En particular, sostiene que: i) el estudio del Derecho puede ser abordado desde diferentes perspectivas, ii) a partir de la existencia de diferentes perspectivas es posible concluir que existen diferentes conceptos de Derecho, y iii) es desde la perspectiva de los participantes en la práctica jurídica —opuesta o diferente a la de los «observadores»- desde la que se puede explicar la verdadera naturaleza del Derecho ${ }^{22}$. Sin embargo, si la reconstrucción ofrecida con anterioridad es correcta —en el sentido de que, para ALEXY, el concepto correcto de Derecho es una idea regulativa subyacente a las convenciones que sustentan los diferentes conceptos vigentes de Derecho-, aunque fuera posible entender la preferencia por uno de los conceptos vigentes de Derecho ${ }^{23}$, no se entiende en qué sentido la verdadera naturaleza del Derecho sólo pueda ser aprehendida desde una perspectiva. Pero antes de concluir que es inconsistente con su concepción acerca de los conceptos considerar que sólo desde la perspectiva de los participantes es posible explicar la naturaleza del Derecho, cabe explorar dónde radica la distinción entre los conceptos de Derecho de participantes y observadores.

En la articulación de su teoría del Derecho, ALEXY dedica un rol central al análisis de la noción de «afirmación», vía la articulación de un argumento que denomina «pragmático-trascendental». No es objeto de este trabajo un estudio pormenorizado de tal tipo de argumentos. Sin embargo, importa precisar de manera breve en qué consisten, para poder comprender de manera general la estrategia explicativa elegida por este autor. En términos generales, los argumentos pragmático-trascendentales estarían constituidos, según AlEXY, al menos, por dos premisas. La primera premisa identifica el punto de partida del argumento, que puede consistir en cosas tales como percepciones, pensamientos o acciones lingüísticas. La segunda premisa, por otro lado, indica cuáles son las reglas o categorías que tienen necesariamente que ser respetadas para que el objeto elegido como punto de partida tenga concreción ${ }^{24}$. ALEXY elige como primera premisa, y, en este sentido, como punto de partida de su argumento pragmático-trascendental el acto lingüístico de afirmar. Lo hace porque considera que la formulación de afirmaciones es parte de la «forma de vida más general de los hom-

22 Cfr. R. Alexy, «An Answer to Joseph Raz», en G. Pavlakos (ed.), Law, Rights and Discourse. The Legal Philosophy of R. Alexy, Oxford, Hart Publishing, 2007, p. 52; R. Alexy, CEV, p. 37. Aquí es posible conjeturar que, AlEXY está pensando en que, desde la perspectiva del participante el concepto de Derecho es sólo uno. Descartaría, en este sentido que, desde la perspectiva del participante puedan ser varios los conceptos de Derecho en juego. Este tiene que ser un presupuesto necesario de su teoría, caso contrario no se explica en qué sentido la perspectiva del participante tiene para el autor privilegio conceptual.

23 En el sentido de que sería comprensible la afirmación de ALEXY que dice que: «Todo aquel que esté interesado en la naturaleza del Derecho, por consiguiente, primero, usará de todos un concepto de Derecho y, segundo, preferirá el concepto que mejor capture sus rasgos esenciales sobre todas las alternativas disponibles», R. ALEXY, «Acerca de dos yuxtaposiciones...», op. cit., pp. 90-91.

${ }_{24}$ Cfr. R. AleXY, «Una concepción teórico discursiva de la razón práctica», en CEVV, pp. 143-144 [título original: «Eine diskurstheoretische Konzeption der praktischen Vernunft», en R. ALEXY y R. DREIER (eds.), Rechtssystem und praktische Vernunft, Archiv für Rechts- und Sozialphiloso-phie, Beiheft 51, 1993, pp. 11-29]. 
bres» ${ }^{25}$, y porque considera al Derecho, cabe agregar, como una empresa estructurada por una práctica lingüística. Luego, en el diferente tipo de afirmaciones que formulan participantes y observadores asienta su argumento para mostrar, por una parte, que participantes y observadores están comprometidos con diferentes conceptos de Derecho; y, por la otra, que el concepto de Derecho con el cual están comprometidos los participantes tiene un privilegio.

Los diferentes tipos de afirmaciones que formulan participantes y observadores queda exhibido, para ALEXY, en las diferentes empresas con las que ambos están comprometidos. Los participantes en la práctica jurídica - a diferencia de los meros observadores- son para ALEXY aquellos que se preguntan y aducen argumentos acerca de lo que está ordenado, prohibido y permitido o autorizado en el contexto del sistema jurídico de referencia, esto es, sobre qué es lo jurídicamente debido. Los participantes, según esta propuesta, son aquellos que se preguntan cuál es la respuesta correcta en el contexto del sistema jurídico de referencia, tanto dentro cuanto fuera del marco institucional ${ }^{26}$. Los observadores, en cambio, son aquellos que se preguntan cómo son de hecho decididas las cuestiones jurídicas en el contexto de un determinado sistema jurídico ${ }^{27}$.

Habiendo elegido como primera premisa, entonces, el acto lingüístico de afirmar, luego especifica aquello que el acto de afirmar necesariamente presupone, de acuerdo con cada perspectiva. Los observadores, al dar respuesta sobre cómo son de hecho decididas las cuestiones jurídicas en el contexto de un sistema jurídico determinado, formulan afirmaciones vinculadas de manera necesaria con una pretensión de verdad. Los participantes, en cambio, al responder acerca de cuáles son las soluciones correctas en el marco de un determinado sistema jurídico, formulan afirmaciones vinculadas de manera necesaria con una pretensión de corrección. Así como una pretensión de verdad es parte de la estructura de los actos de habla afirmativos de los observadores, cuyo objeto son enunciados no normativos, una pretensión de corrección es parte de la estructura de los actos de habla afirmativos de los participantes, cuyo objeto son enunciados normativos. Esta diferencia en el compromiso pragmático de las afirmaciones de observadores y participantes no siempre es puesta de manifiesto por ALEXY.

25 La primera premisa del argumento pragmático-trascendental que estructura su teoría dice: «Quien a lo largo de su vida no plantea ninguna afirmación seria y no da ninguna fundamentación seria, no toma parte en lo que podría llamarse "forma de vida más general de los hombres"», R. ALEXY, Teoría de la argumentación jurídica, trad. M. AtiEnZA e I. EsPejo, Madrid, Centro de Estudios Constitucionales, 1989, pp. 305-306 (título original: Theorie der juristischen Argumentation. Die Theorie des rationalen Diskurses als Theorie der juristischen Begründung, Frankfurt a/M, Suhrkamp, 1983; en adelante citaré la versión en castellano como TAJ).

${ }_{26}$ Cfr. R. AleXY, «An Answer to Joseph Raz», op. cit., p. 47. Para terminar de delinear esta perspectiva, es posible añadir que, para ALEXY, la posición paradigmática de participante es la del juez o legislador; y que juristas, abogados y ciudadanos cuando argumentan acerca de lo ordenado, prohibido y permitido, lo hacen tomando como referencia su posición. Cfr. R. ALEXY, CEV, p. 31.

27 ALEXY asimila el concepto de los observadores a un concepto positivista del Derecho y el concepto de los participantes a un concepto antipositivista del Derecho. Cfr. R. AlEXY, «An Answer to Joseph Raz», op. cit., pp. 45-48; R. Alexy, CEV, p. 31; R. Alexy, «On Necessary Relations Between Law and Morality», Ratio Juris, vol. 2, julio 1989, p. 171. Si se acepta la objeción que en este trabajo planteo a ALEXY, con relación a su presentación de la empresa con la cual todo observador está comprometido, será posible comprender por qué el acierto de tal equiparación puede ser puesta en duda. En otro orden, es posible añadir que, ALEXY reconoce una similitud — aunque no una identidad - entre las nociones de «participantes» y «observadores» y las de «punto de vista interno» $\mathrm{y}$ «punto de vista externo» de H. HART. 
Sin embargo, un compromiso con esta distinción es necesario, si lo que se busca es dar sentido a la diferencia que traza entre las empresas de participantes y observadores. En cualquier caso, quienes desde una posición de observadores o participantes nieguen, respectivamente, la pretensión de verdad o la pretensión de corrección implícitas de manera necesaria en sus afirmaciones, incurrirían en una contradicción preformativa; esto es, una contradicción entre lo que se dice y lo que está implícito de manera necesaria en el acto de decir ${ }^{28}$.

Considero relevante tener en cuenta la diferente naturaleza de las afirmaciones mencionadas ya que, es la articulación de los diferentes compromisos pragmáticos implicados en cada una de ellas lo que lleva a ALEXY a considerar que existen distintos conceptos de Derecho, que conducen a un conocimiento de la naturaleza del Derecho total o parcial ${ }^{29}$. La tesis que dice que participantes y observadores están necesariamente comprometidos con diferentes tipos de afirmaciones está presupuesta por ALEXY, ya que el autor cifra en el análisis de los compromisos pragmáticos de las afirmaciones de los participantes, y no en la de los observadores, la clave en la explicación de la naturaleza del Derecho. En este trabajo tal asunción no será puesta en cuestión. Lo que sí intentaré mostrar, como se verá, es la inconsistencia en la que ALEXY incurre al sostener que, desde la perspectiva de los observadores una explicación de la verdadera naturaleza del Derecho es inalcanzable. Para ello es necesario aún expandir su argumento.

De acuerdo con las distinciones trazadas, es posible notar la manera en que la noción de pretensión de corrección deviene una pieza central en su teoría del Derecho. ALEXY, al afirmar que sólo desde la perspectiva de los participantes es aprehensible la verdadera naturaleza del Derecho, convierte a la noción de pretensión de corrección en la llave para el acceso al mundo jurídico como mundo normativo. Según este autor, la noción de pretensión de corrección es lo que permite comprender a la noción de «deber» como algo diferente de la noción de «querer» ${ }^{30}$. En este sentido, considera imprescindible el análisis de la noción de pretensión de corrección para mostrar el sentido en que el Derecho difiere del mero poder. Dado que entiende que el Derecho no puede ser reducido al ejercicio desnudo de poder, considera central la articulación de los compromisos pragmáticos de las afirmaciones de los participantes. Se podría así señalar que el privilegio conceptual de los participantes presupone que el Derecho tiene una dimensión normativa. Si esto es así, aun cuando se admita que tal dimensión normativa sólo es aprehensible desde la perspectiva de los participantes, todavía sería posible cuestionar que una explicación de tal dimensión sea relevante en la explicación de la naturaleza del Derecho. AleXY considera que tal alternativa es equivocada, y no es mi intención profundizar aquí sobre este punto. Aquí lo que importa es retener que, para ALEXY, la forma en que los participantes entienden a sus propias prácticas no está separada de lo que estas prácticas son. Lo que está en cuestión, es oportuno subrayarlo, son los compromisos conceptuales de los participantes, no la posesión más

28 ALEXY sostiene que, en este caso, se podría hablar de una «falla conceptual» en sentido amplio. Esto si se entiende a la noción de «falla conceptual» como aquella violación de las reglas constitutivas de los actos lingüísticos - esto es, de las expresiones lingüísticas como acciones-. Cfr. R. ALEXY, CEVV, p. 43; R. ALEXY, NANL, p. 12; R. ALEXY, ONRBLEM, pp. 178 y ss.

${ }^{29}$ Cfr. R. AleXY, «An Answer to Joseph Raz», op. cit., p. 52.

${ }^{30}$ Cfr. R. Alexy, NANL, p. 13. 
o menos perfecta que de tal concepto se tenga, o el uso más o menos perfecto que de tal concepto se haga.

Lo que aún queda por elucidar es si ALEXY, de manera consistente, puede afirmar que sólo desde la perspectiva de los participantes se puede aprehender la naturaleza del Derecho. AleXY intenta mostrar que éste es el caso a partir de una afirmación que, sostiene, sería contradictoria si fuera sostenida por un observador, pero no así por un participante. La afirmación en cuestión dice:

«A no ha sido privado de su nacionalidad de acuerdo con el Derecho alemán a pesar de que todos los tribunales y autoridades alemanes tratan a $A$ como privado de su nacionalidad y se apoyan para ello en el texto de una norma que, de acuerdo con los criterios de validez del sistema jurídico vigente en Alemania, ha sido promulgada correctamente» ${ }^{31}$.

Si bien la afirmación podría ser sostenida sin contradicción por un participante, señala ALEXY, habría una contradicción si el que la profiere es un observador. La razón que, según AlEXY, justifica tal diferencia, es que observadores y participantes están comprometidos con diferentes conceptos de Derecho. Ello llevaría a que, mientras que a los observadores sólo les es accesible la dimensión autoritativa o fáctica del Derecho, a los participantes también les es accesible su dimensión ideal, que es lo que les permitiría sostener sin contradicción la afirmación citada. Con ello, lo que ALEXY quiere resaltar es que desde la perspectiva de los observadores no se puede afirmar que: i) «A no ha sido privado de su nacionalidad de acuerdo con el Derecho alemán», si, a su vez: ii) «todos los tribunales y autoridades tratan a $A$ como privado de su nacionalidad, y se apoyan para ello en el texto de una norma que, de acuerdo con los criterios de validez del sistema jurídico vigente en Alemania, ha sido promulgada correctamente», dado que ii) es lo único que puede ser dicho por el observador, teniendo en cuenta su concepto de Derecho. Pero AleXY, al decir esto, pareciera olvidar tanto su definición de «observador» cuanto su propuesta según la cual todo concepto pretende adecuarse a su objeto.

La afirmación a la que ALEXY alude nunca podría ser sostenida por un observador, en virtud de su propia definición de «observador». Si se recuerda que según su concepción los observadores son aquellos que se preguntan sobre cómo son de hecho decididas las cuestiones jurídicas, mientras que los participantes son aquellos que se preguntan por cuál es la respuesta correcta, se advierte que la afirmación referida por ALEXY sólo puede ser sostenida por un participante (por definición) ${ }^{32}$. De manera tal que la contradicción a la que hace referencia ALEXY nunca podría verificarse. Por otro lado, si la naturaleza del Derecho es sólo una, y todo concepto de Derecho vigente tiene incorporada de manera necesaria una pretensión de adecuación a esa naturaleza, ¿por qué concluir que sólo desde la perspectiva de los participantes es posible acceder a la verdadera naturaleza del Derecho?

Afirmar que del análisis del esquema conceptual de los observadores no se desprende un compromiso con ningún valor, no implica que parte de su empresa no pueda consistir, por un impedimento conceptual, en la descripción de un valor con el que otros sí están comprometidos, y que es lo que explica la idea de Derecho como algo

31 Cfr. R. Alexy, CEV , pp. 35-36; R. AleXY, «An Answer to Joseph Raz», op. cit., p. 47.

32 Quiero agradecer a C. REDONDO por haberme hecho ver este punto. 
diferente a la idea de mero poder ${ }^{33}$. Si esto no fuera cierto, ALEXY tiene que concluir que el concepto de los observadores se refiere a otra cosa, a algo que no es Derecho. Lo que intento poner de manifiesto es que ALEXY no puede decir de manera consistente que existen dos conceptos de Derecho que no pretendan adecuarse a la verdadera naturaleza del Derecho, si se tiene en cuenta la noción de «concepto» de la cual parte. En este sentido, si el concepto correcto de Derecho fija un criterio a partir del cual pensar la naturaleza de las prácticas jurídicas, en un manejo acabado del mismo tienen que coincidir participantes y observadores. Caso contrario tendrán conceptos sobre distintas $\operatorname{cosas}^{34}$.

\section{REFLEXIONES FINALES}

Finalmente, luego de las precisiones teóricas trazadas surge una nueva pregunta: si la naturaleza del Derecho sólo se expresa en el concepto correcto de Derecho, ¿̇en qué sentido el estudio de la naturaleza del Derecho es la tarea prioritaria del teórico del Derecho, y el análisis del concepto de Derecho tan sólo una parte de tal tarea? Si la lectura de las tesis de ALEXY que ofrezco en este trabajo es correcta, hay que concluir que desde su concepción teórica objeto y concepto correcto de Derecho son interdependientes, ya que la naturaleza del Derecho sólo alcanza su expresión en el concepto correcto de Derecho que la refleja. En este sentido, el análisis del concepto correcto de Derecho agota la tarea filosófica.

33 Cosa que, por otra parte, el mismo Alexy advierte en R. Alexy, CEV, pp. 40-41.

34 Ello, a su vez, haría que afirmar que hay tesis (positivistas o antipositivistas) acerca de la naturaleza del Derecho que son válidas desde la perspectiva del observador e inválidas desde la perspectiva del participante, o viceversa, resulte falsa. Bajo la propuesta meta-teórica ofrecida por ALEXY, la validez o invalidez de las tesis referidas puede predicarse en función de su ajuste o desajuste a la naturaleza del Derecho, que es única, y que no podría variar según sea la perspectiva elegida. ALEXY, por el contrario, sostiene que, por ejemplo, la tesis de la separación entre Derecho y moral es válida desde la perspectiva del observador e inválida desde la perspectiva del participante. Ello sería, según lo aquí señalado, inconsistente con sus propios compromisos metateóricos. Cfr. R. AleXY, CEV , pp. 33 y ss. Una crítica diferente a la idea de que participantes y observadores tienen diferentes conceptos de Derecho puede encontrarse en J. RAZ, «The Argument from Injustice or How not to Reply to Legal Positivism», en G. Pavlakos (ed.), Law, Rights and Discourse. The Legal Philosophy of R. Alexy, op. cit., pp. 22-25. 